\title{
Nonlinear Optical Frequency Conversion with KTP and BiBO Crystals for Lasers in Space
}

Arne Potreck ${ }^{\mathrm{a}}$, Helmut Schröder*a, Melanie Lammers ${ }^{\mathrm{a}}$, Georgios Tzeremes ${ }^{\mathrm{b}}$, Wolfgang Riede ${ }^{\mathrm{a}}$ ${ }^{a}$ Institute of Technical Physics, German Aerospace Center, Pfaffenwaldring 38, D-70569 Stuttgart, Germany; ${ }^{b}$ European Space Agency, ESTEC, P.O. Box 299, 2200AG, The Netherlands

\begin{abstract}
Within ESA's ADM-Aeolus and EarthCARE missions Doppler-wind Lidar systems will be operated in the Earth's orbit to measure global wind profiles. The active instrument will be based on a Nd:YAG laser, frequency tripled by nonlinear optical crystals. Different crystals are therefore to compare and qualify in regard of their space acceptability. A dedicated set-up to measure the maximum conversion efficiencies and its stability during longterm operation for KTP crystals (SHG) and BiBO crystals (SHG and THG) is presented in this work. In order to detect gray-tracking and its influence on thermal lensing in situ, measurements with a Shack-Hartmann sensor and a co-aligned HeNe laser were performed. Conversion efficiencies were $76 \pm 3 \%$ at SHG for KTP and BiBO crystals and $48 \pm 2 \%$ at THG with a combination of two BiBO crystals. During longterm experiments of 60 million laser pulses, conversion efficiencies were demonstrated to be stable over time $( \pm 1 \%$ at SHG with KTP and $\pm 2 \%$ at THG with BiBO). The occurrence of gray-tracking was detected in the KTP crystal and the resulting thermal lensing with an exponential saturation over time was observed in situ.
\end{abstract}

Keywords: gray-tracking, thermal lensing, KTP, BiBO, ADM-Aeolus

\section{INTRODUCTION}

ESA's Atmospheric Dynamics Mission (ADM)-Aeolus aims to gain information on global vertical wind profiles by operating a Lidar system in the Earth's orbit. The satellite's active instrument consists of a high power laser emitting laser pulses towards the atmosphere. From the Doppler shift of the backscattered light and the pulses propagation time, vertical wind profiles will be determined ${ }^{1}$. Onboard the satellite, a frequency converted Nd:YAG laser will be operated ${ }^{1}$. For the frequency conversion two LBO crystals are planned ${ }^{2}$. However, the nonlinear crystals Bismuth Triborate (BiBO) and Potassium Titanyl Phosphate (KTP) show higher effective nonlinearities and therefore could be promising candidates for future space applications. On the other hand, these crystals have some drawbacks when compared to LBO crystals. During longterm operation, gray-tracking can occur in KTP crystals. The changing absorption might influence the conversion efficiency and lead to self-heating and consequently to thermal lensing. A drawback in BiBO crystals could be the smaller temperature and angular acceptance bandwidths when compared to LBO and KTP crystals (Table 1). Therefore, the maximum conversion efficiencies for KTP crystals (for second harmonic generation) and BiBO crystals (second and third harmonic generation) were determined. To verify, if gray-tracking has occurred, indirect measurements of the thermal lens were performed. Longterm stability of the conversion efficiency and the thermal lens was investigated with an experiment over 60 million laser pulses, which is approximately $1 \%$ of the mission's lifetime.

Table 1. Crystals under investigation ${ }^{3}$; dimension of the crystal facets: $10 \times 10 \mathrm{~mm}^{2}$; a flux-grown anti-gray-tracking $\mathrm{KTP}_{\mathrm{gr}}$ crystal was used for the experiments.

\begin{tabular}{ccccccc}
\hline Crystal & Length $[\mathrm{mm}]$ & $\begin{array}{c}\text { Phase } \\
\text { matching type }\end{array}$ & $\begin{array}{c}\mathrm{d}_{\text {eff }} \\
{[\mathrm{pm} / \mathrm{V}]}\end{array}$ & $\begin{array}{c}\text { Walk-off angle } \\
{[\mathrm{mrad}]}\end{array}$ & $\begin{array}{c}\Delta \Theta / \Delta \Phi[\mathrm{deg}] \\
\mathrm{L}=1 \mathrm{~cm}\end{array}$ & $\begin{array}{c}\text { Temp. bandwidth } \\
{[\mathrm{Kcm}]}\end{array}$ \\
\hline $\mathrm{KTP}_{\mathrm{gr}}(\mathrm{SHG})$ & 9.0 & $\mathrm{II}$ & 3.56 & 4.28 & $0.58 / 1.82$ & 20 \\
\hline BiBO (SHG) & 10.4 & $\mathrm{I}$ & 3.00 & 27.55 & 0.04 & 1.3 \\
\hline BiBO (THG) & 10.0 & I & 3.9 & 68.6 & $0.051 / 0.024$ & 0.9 \\
\hline LBO $(\mathrm{SHG})$ & - & I & 0.836 & 6.03 & $0.27 / 2.63$ & $5.8-6.7$ \\
\hline LBO (THG) & - & II & 0.52 & 9.5 & $0.79 / 0.16$ & 6 \\
\hline
\end{tabular}

*corresponding author: helmut.schroeder@dlr.de; phone +49 7116862537 


\section{MATERIAL AND METHODS}

\subsection{Experimental set-up}

The laser pulses (Coherent Infinity 40-100 with 3 ns pulse width at $100 \mathrm{~Hz}$ ) pass the frequency conversion unit and are split into the fundamental and generated harmonics (see Figure 1). The crystals were mounted in a holder suitable for the planned spaceborne application. It is made of oxygen-free high thermal conductivity (OFHC)-copper, mounted on a Macor base-plate. To reduce radiative losses, a thin gold coating was applied to the holder. By wrapping the crystals in a thin indium foil, the thermal contact between crystal and mount was improved. With an infrared camera (Flir T-420), the spatial and temporal homogeneity of the crystal's temperature was determined to be $0.1 \mathrm{~K}$. As listed in Table 1 , the most demanding situation was found at THG with a $10 \mathrm{~mm}$ long BiBO crystal, having a FWHM temperature bandwidth of only $0.9 \mathrm{~K}$. Here, the temperature homogeneity allows stable frequency conversion with variations of less than $3.5 \%$ (when assuming a sinc ${ }^{2}$-dependency of the conversion efficiency on the temperature).

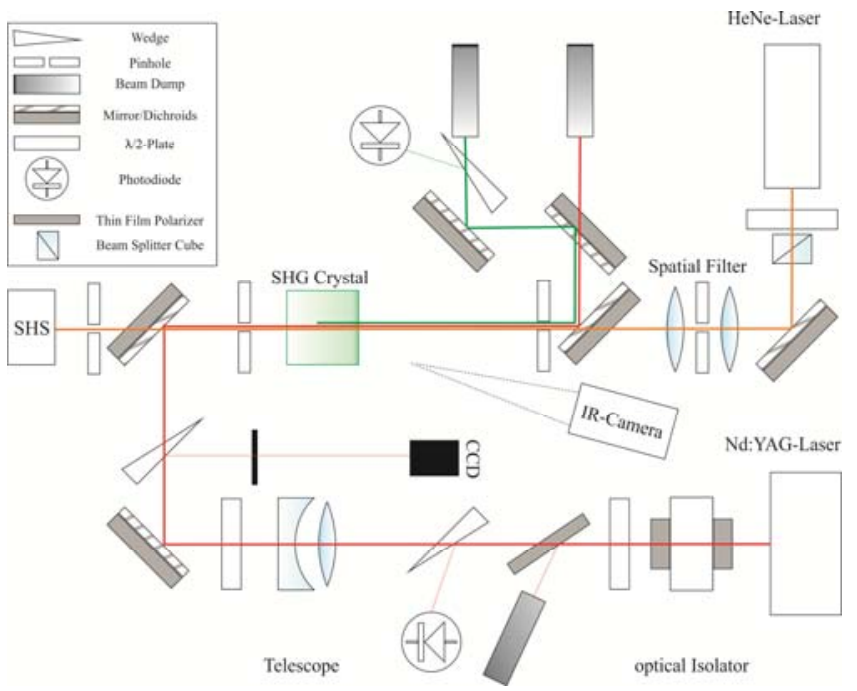

Figure 1. Set-up for frequency conversion experiments; a co-aligned but antiparallel HeNe laser allows measuring the wavefront deformation, induced by absorption of the generated harmonic. With an IR camera, the temperature distribution on the crystal's exit facet was measured ${ }^{4}$.

\subsection{Thermal lensing}

A HeNe laser was coaligned to the Nd:YAG laser and the thermal lens determined by measuring the resulting wavefront deformation with a Shack-Hartmann sensor (Haso 3-32). Since thermal lensing is driven by the absorption, this method allows gaining information on absorption as well as on the resulting focusing of the beam in situ. The measured wavefront depends on the optical path length differences $\Delta \tau$, which the HeNe laser experiences in the crystal. If heating is not homogenous, e.g. because of the Gaussian beam profile of the Nd:YAG laser, a wavefront deformation results. Optical path length changes $\Delta \tau$ are caused by the temperature dependent change in the refractive index $\Delta \mathrm{n}_{\mathrm{T}}(\delta \mathrm{T})$, by the linear thermal expansion $\kappa$ of the crystal and by changes in the refractive index due to strain $\Delta \mathrm{n}_{\text {strain }}(\delta \mathrm{T})^{5}$. The optical path length difference, resulting from the propagation through the crystal is therefore given by:

$$
\Delta \tau=\int d z\left(\Delta \mathrm{n}_{\mathrm{T}}(\delta \mathrm{T})+n \kappa \delta T(z)+\Delta n_{\text {strain }}(\delta T)\right)
$$

Like Mansell et al. ${ }^{5}$, we defined the magnitude of the thermal lens $\Delta \tau_{\text {mag }}$, to be the optical path length difference between the center of the beam $(\mathrm{r}=0)$ and the $1 / \mathrm{e}^{2}$-beamwidth $(\mathrm{r}=\omega)$ :

$$
\Delta \tau_{\text {mag }}=|\Delta \tau(0)-\Delta \tau(\omega)|
$$

This allows to describe the thermal lens and to monitor its changing over time in the following. Additionally, the temperature distributions on the exit facets of the crystals were monitored using the infrared camera. 


\subsection{Longterm experiments}

The crystals were typically exposed to 60 million laser pulses (corresponding to one percent of the mission's lifetime) and conversion efficiency as well as thermal lensing was monitored. A thermal lens in the crystal, which is stable over time, might be of minor interest for spaceborne application. In contrast to this, if thermal lensing is not constant (e.g. because of changes in absorption due to gray-tracking), the transmitted beam's divergence changes.

\section{RESULTS}

Maximum conversion efficiency at SHG was $76 \pm 3 \%$ (at a fluence of $2.50 \pm 0.25 \mathrm{~J} / \mathrm{cm}^{2}$ ) with the KTP crystal and also $76 \pm 3 \%$ (at a fluence of $1.66 \pm 0.15 \mathrm{~J} / \mathrm{cm}^{2}$ ) with the BiBO crystal. For the KTP crystal, a Gaussian like temperature distribution with a maximum amplitude of $+5.0 \pm 0.2{ }^{\circ} \mathrm{C}$ was found, whereas the temperature was homogenous on the BiBO crystal's exit facet $\left( \pm 0.1^{\circ} \mathrm{C}\right)$. Also when measuring the wavefront deformation, no relevant thermal lensing was measured in the BiBO crystal within measurement accuracy $( \pm 7 \mathrm{~nm})$, whereas a $\Delta \tau_{\text {mag }}$ of $163 \pm 5 \mathrm{~nm}$ was found in the KTP crystal.

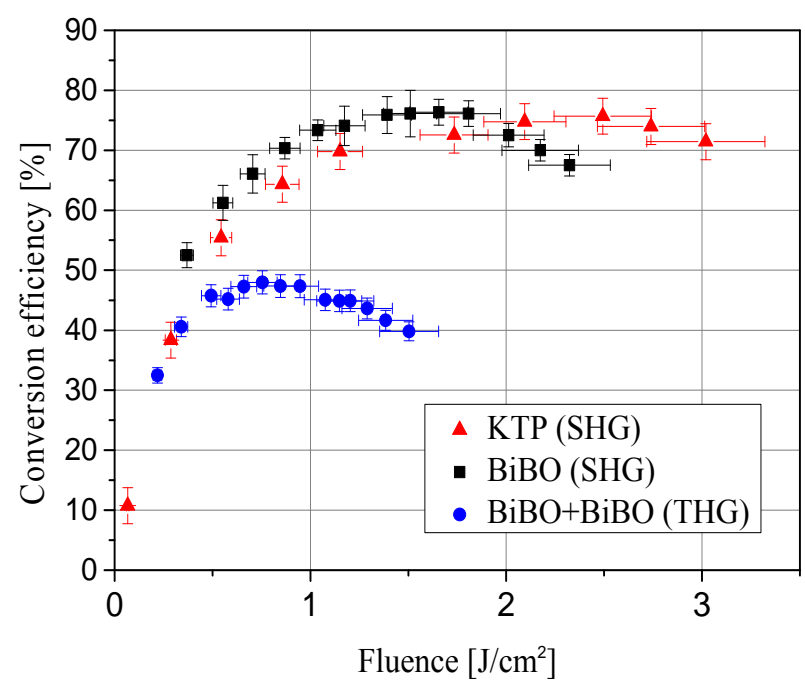

Figure 2. Measured conversion efficiency versus entrance fluence for second harmonic generation with KTP and BiBO crystals and for third harmonic generation with $\mathrm{BiBO}$ crystals $^{4}$.

Conversion efficiency during longterm operation at SHG with the BiBO crystal was $69 \pm 1 \%$ (at a fluence of $1.6 \pm 0.2 \mathrm{~J} / \mathrm{cm}^{2}$ ). It did not show any systematic decline over time. With the KTP crystal, the conversion efficiency was found to decrease over the first 10 million pulses from $67 \%$ to $63 \%$ and stabilized afterwards at $64 \pm 1 \%$ (at a fluence of $2.4 \pm 0.3 \mathrm{~J} / \mathrm{cm}^{2}$ ). The temperature distribution on the exit facets of both crystals showed no tendencies or significant variations over time. In contrast to this, the optical path length difference $\Delta \tau_{\text {mag }}$ increased over time in the KTP crystal. It started at $160 \pm 5 \mathrm{~nm}$ and asymptotically reached $193 \pm 4 \mathrm{~nm}$, with a half-life time of 11 million laser shots (Figure 3 ), thus focusing of the beam increased over time. Since gray-tracking anneals when the crystals are exposed to higher temperatures, the KTP crystal was heated to $125^{\circ} \mathrm{C}$ for 24 hours. Afterwards, the thermal lens was found to return to the initial value at $155 \pm 7 \mathrm{~nm}$. In the BiBO crystal, $\Delta \tau_{\text {mag }}$ was found to be (within measurement accuracy) stable over the longterm operation $(15 \pm 5 \mathrm{~nm})$.

At third harmonic generation with the two BiBO crystals, the maximum conversion efficiency was realized at a fluence of $0.75 \pm 0.08 \mathrm{~J} / \mathrm{cm}^{2}$ and an efficiency of $48 \pm 2 \%$ (Figure 2). Due to the higher absorption in the ultraviolet, a wavefront deformation was now observed in $\mathrm{BiBO}$ as well $\left(\Delta \tau_{\mathrm{mag}}\right.$ of $\left.79 \pm 16 \mathrm{~nm}\right)$. Also the temperature gradient on the exit facet of the THG crystal had an amplitude of $0.9 \pm 0.1 \mathrm{~K}$. During longterm operation, the conversion efficiency was $35 \pm 2 \%$ (at a fluence of $1.4 \pm 0.2 \mathrm{~J} / \mathrm{cm}^{2}$ ), showing no systematic decrease or changes over time. Also $\Delta \tau_{\text {mag }}$ stabilized at $120 \pm 20 \mathrm{~nm}$. A fast growing in $\Delta \tau_{\text {mag }}$ during the first 0.5 million pulses represented the stabilization of the thermal lens. Also here, the temperature distribution on the exit facet of the THG crystal was constant over time $\left(+1.0 \pm 0.3^{\circ} \mathrm{C}\right)^{4}$. 


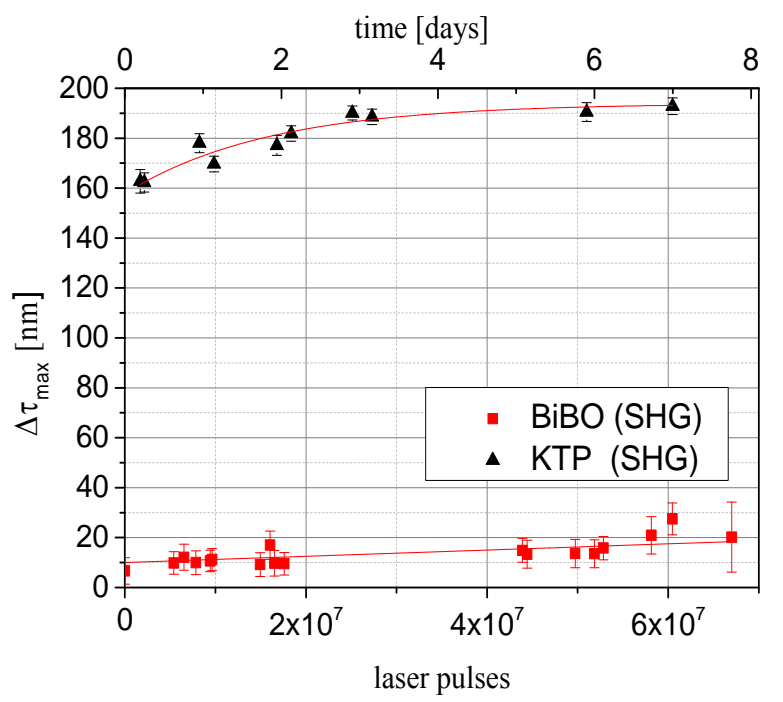

Figure 3. $\Delta \tau_{\text {mag }}$ during longterm operation at second harmonic generation with KTP and BiBO crystals. No significant thermal lensing was detected in $\mathrm{BiBO}$ crystal, whereas $\Delta \tau_{\text {mag }}$ changed over time in the KTP crystal ${ }^{4}$.

\section{CONCLUSION}

Conversion efficiencies of $76 \pm 3 \%$ (at SHG) with KTP and BiBO crystals, and of $48 \pm 2 \%$ (at THG) with a combination of two BiBO crystals were realized. The efficiency was demonstrated to be stable during longterm operation $( \pm 1 \%$ at SHG with KTP and $\pm 2 \%$ at THG with BiBO). Additionally, the occurrence of gray-tracking was detected in the KTP crystal and the resulting changes in thermal lensing with an exponential saturation over time could be observed in situ. Its influence on conversion efficiency or on the temperature distribution on the crystal's exit facet was below measurement threshold ${ }^{4}$.

\section{ACKNOWLDGMENT}

The work is performed with kind support by ESA/ESTEC under contract: 4000104824/11/NL/PA. A. Potreck gratefully acknowledges the support by the High-Energy Astrophysics Group at the Institute of Astronomy and Astrophysics of the University of Tübingen, Germany (IAAT).

\section{REFERENCES}

[1] Clissold, P., Andersson, E., Dabas, A., Endemann, M., Ingmann, P., Källén, E., Offiler, D. and Stoffelen, A.,[ADMAeolus Science Report SP-1311]. ESA, ESTEC, Noordwijk, The Netherlands ( 2008).

[2] Endemann, M., "The ADM-Aeolus Mission," Proc. 6th Internat. Conf. on Space Optics, (2006).

[3] Nikogosyan, D. N., [Nonlinear Optical Crystals], Springer, Berlin \& Heidelberg \& New York, 54-74, 215-218 (2005).

[4] Potreck, A. "Nonlinear Optical Frequency Conversion for Lasers in Space," Diploma thesis, Institute of Astronomy and Astrophysics of the University of Tübingen (2014).

[5] Mansell, J. D., Hennawi, J., Gustafson, E. K., Fejer, M. M., Byer, R. L., Clubley, D., Yoshida, S. and Reitze, D. H., "Evaluating the effect of transmissive optic thermal lensing on laser beam quality with a Shack-Hartmann wavefront sensor," Appl. Opt., 40(3),366-374 (2001). 